\title{
THE ENVIRONMENTAL CONDITION (LIGHT POLLUTION AND RADIO INTERFERENCE) OF CHINESE ASTRONOMICAL OBSERVATORIES, PAST, PRESENT, AND FUTURE
}

\section{JIANG SHI YANG}

Beijing Astronomical Observatory, Beijing, PRC

\begin{abstract}
Although China is a backward developing country, the population of many big cities has increased more than 100 percent since 1950. The light and smoke pollution for Shanghai Observatory and Purple Mountain Observatory is very serious, and in Kunming, for Yunnan Observatory, is also quite a problem. Now the only one where the observational condition is still quite good is the optical station of the Beijing Observatory, in XingLong County, Hebei Province. For the future, we plan to do some testing work on the Tibet plateau, where the population if very low, so there is no light pollution at all.

Concerning radio interference for our major observatories, we also have to do some testing in limited areas and limited frequency bands. The situation at Miyun Station of the Beijing Observatory is quite good, as well as at the Delinha microwave station of the Purple Mountain Observatory. The mean disturbing level is less than $10 \mathrm{dbm}$ in CISPR standard. For the future, we have to make some agreement with the local government to limit the radio disturbing sources in the surrounding area of our observational stations.
\end{abstract}

